\title{
CLOSURE, CONTRAST, AND ANSWER
}

\begin{abstract}
How should the contrastivist formulate closure? That is, given that knowledge is a ternary contrastive state $\mathrm{K} s p q$ ( $s$ knows that $p$ rather than $q$ ), how does this state extend under entailment? In what follows, I will identify adequacy conditions for closure, criticize the extant invariantist and contextualist closure schemas, and provide a contrastive schema based on the idea of extending answers. I will conclude that only the contrastivist can adequately formulate closure.
\end{abstract}

\section{CONTRASTIVISM AND CLOSURE}

There are two main assumptions I will be making that should be flagged from the start. First, I will be assuming a contrastive theory of knowledge. ${ }^{1}$ That is, I will be assuming that knowledge is a ternary contrastive state $\mathrm{K} s p q$ (where $s$ is the subject, $p$ is the proposition known, and $q$ is the contrast proposition), whose evidential component Espq is the elimination of the $q$-worlds. This assumption will not be directly defended here. Though what follows may be considered an indirect defense, insofar as contrastivism proves needed for an adequate formulation of closure.

Second, I will be assuming that some form of closure holds. After all, (i) the idea that knowledge extends under entailment is intuitively compelling (Feldman, 1995); (ii) mathematical knowledge, as based on proof, presupposes such extension (Williamson, 2000); and (iii) denying closure allows abominable conjunctions such as "I do not know that I am not a (handless) brain-in-a-vat, but I know that I have hands" (DeRose, 1995). This assumption will not be further defended here. ${ }^{2}$ Though what follows may be considered an indirect defense, insofar as contrastive closure proves safe from objectionable consequences. 
Putting this together, I will be assuming:

(A1) Contrastivism: The knowledge state is Kspq, where $q$ is a contrast proposition, and whose evidential component Espq is the elimination of the $q$-worlds.

(A2) Closure: Kspq satisfies some closure schema.

So the question becomes, what closure schema holds for the contrastive state Kspq?

\section{BINARY CLOSURE}

It will prove useful to begin with closure schemata for binary Ksp. This will help identify adequacy conditions, illustrate some useful maneuvers, and reveal problems with binarity.

The simplest binary closure schema runs:

$$
\left(\mathrm{K} s p_{1} \&\left(p_{1} \rightarrow p_{2}\right)\right) \rightarrow \mathrm{K} s p_{2}
$$

In words: if $s$ knows that $p_{1}$ and $p_{1}$ entails $p_{2}$, then $s$ knows that $p_{2}$. In short, knowledge is closed under entailment.

What can be said for 1 is that it preserves the intuitions behind closure. That is, it provides a plausible rendering of how knowledge extends under entailment, explains how proof extends knowledge, and blocks abominable conjunctions. These make 1 a worthy candidate to be a closure schema. This yields three adequacy conditions on closure:

(C1) An adequate closure schema should provide a plausible rendering of how knowledge extends under entailment.

(C2) An adequate closure schema should explain how proof extends knowledge.

(C3) An adequate closure schema should block abominable conjunctions. To fail $\mathrm{C} 1-\mathrm{C} 3$ is to be unsuited for the role of closure.

What can be said against 1 is that it faces counterexamples. First, $s$ might fail to see some consequences of what he knows. We are not logically omniscient. Second, $s$ might see some consequence but still fail to believe it. Third, $s$ might 
see and believe some consequence, but on the wrong basis. Perhaps $s$ only forms his belief from reading tea leaves. Thus 1 is false. This yields three further adequacy conditions:

(C4) An adequate closure schema should block lack-ofomniscience counterexamples.

(C5) An adequate closure schema should block lack-ofbelief counterexamples.

(C6) An adequate closure schema should block irrationalbasis counterexamples.

To fail $\mathrm{C} 4-\mathrm{C} 6$ is to be inapplicable to human knowledge.

An improved binary closure schema runs:

(2) $\quad\left(\mathrm{K} s p_{1} \& \mathrm{~K} s\left(p_{1} \rightarrow p_{2}\right)\right) \rightarrow \mathrm{K} s p_{2}$

In words: if $s$ knows that $p_{1}$ and $s$ knows that $p_{1}$ entails $p_{2}$, then $s$ knows that $p_{2}$. In short, knowledge is closed under known entailment. ${ }^{3}$

What can be said for 2 is that it satisfies $\mathrm{C} 1-\mathrm{C} 4$. That is, 2 preserves the virtues of 1 with respect to $\mathrm{C} 1-\mathrm{C} 3{ }^{4}$ And by requiring that the entailment be known, 2 blocks lack-ofomniscience counterexamples, thus satisfying $\mathrm{C} 4$. But what can be said against 2 is that is fails $\mathrm{C} 5-\mathrm{C} 6$. That is, 2 remains subject to counterexample in cases where $s$ fails to believe that $p_{2}$, or $s$ believes that $p_{2}$ on an irrational basis (perhaps by ignoring the proof in favor of trusting the tea leaves).

Perhaps the best binary closure schema revises the consequent of 1, replacing $\mathrm{K} s p_{2}$ with $\mathrm{E} s p_{2}$, where $\mathrm{E}$ is the evidential component of $\mathrm{K}$ :

$$
\left(\mathrm{K} s p_{1}\left(p_{1} \rightarrow p_{2}\right)\right) \rightarrow \mathrm{Esp}_{2}
$$

That is, position to know is closed under entailment, where position to know is understood as satisfying the evidential component of knowledge. So if Esp $p_{2}$, then $s$ has propositional justification for $p_{2}$, and is perhaps only a belief-based-oncompetent-deduction-while-retaining-knowledge-of- $p_{1}$ away from $\mathrm{K} s p_{2}{ }^{5}$

What can be said for 3 is that is satisfies C1-C6. Essentially, 3 preserves the virtues of 1 with respect to $\mathrm{C} 1-\mathrm{C} 3$. And 
3 satisfies $\mathrm{C} 4-\mathrm{C} 6$ because failures of omniscience, belief, and basing are no impediment to $\mathrm{E}$. Thus the progression from 1 to 3 reveals how to surmount the omniscience, belief, and basing problems. The binary theorist might well rest here.

What can be said against 3 (and binary closure generally) is that it engenders skeptical paradox. To begin with, 3 is incompatible with A1. 3 is formulated for $\mathrm{K} s p$, not for the contrastive Kspq. Of course, the binary theorist will hardly flinch. But A1 is an assumption of the current discussion ( $(1)$.

Further, 3 precludes epistemic modesty. Surely we possess modest knowledge of the external world. For instance, Moore knows that he has hands. He need only look. And surely we also suffer modest ignorance of the external world. For instance, Moore does not know that he is not a brain-in-avat. He may look all he wants, but it won't help. But 3 forces our modest knowledge and modest ignorance into conflict, driving us to dogmatism or skepticism. ${ }^{6}$

Finally, 3 mistreats knowledge-whether. Intuitively, Moore knows whether he has hands or stumps. As to whether he has hands or stumps, he can tell by looking. This is an easy question. And intuitively, Moore does not know whether he has hands or vat-images of hands. As to whether he has hands or vat-images of hands, he can't tell - they'd look the same to him. This is an (impossibly) difficult question.

Yet 3, together with the standard binary view of knowledge-whether, forces these intuitive claims into conflict. The standard binary view of knowledge-whether reduces it to $\mathrm{K} s p$, where $p$ is the true answer to the whether-question. ${ }^{7}$ So (given that Moore in fact has hands), his knowledge of whether he has hands or stumps is his knowledge that he has hands. This alleged knowledge would position Moore to know that he is not a brain-in-a-vat. This would position him to know whether he has hands or is a brain-in-a-vat, which of course he is in no position to know.

This yields three further adequacy conditions:

(C7) An adequate closure schema should concern the contrastive Kspq state. 
(C8) An adequate closure schema should preserve epistemic modesty.

(C9) An adequate closure schema should fit knowledgewhether.

C7-C9 are connected. Moore's knowledge as to whether he has hands or stumps, and his ignorance as to whether he has hands or vat-images of hands, comprises his modest epistemic standing. There are some questions he can answer, and some he can't. This modest standing is expressible by going explicit about the contrasts: Moore knows that he has hands rather than stumps, but Moore does not know that he has hands rather than vat-images of hands. Here is the path between dogmatism and skepticism. To fail C7-C9 is to fall into skeptical paradox.

\section{CONTEXTUALIST CLOSURE}

It will prove useful to turn to the standard contextualist formulations of closure, since contextualism and contrastivism are close relatives. This will help identify further adequacy conditions for closure, illustrate more useful ideas, and reveal problems for the contextualist.

The standard contextualist view is that closure holds as long as the relevant alternatives are fixed. Here is the simplest meta-linguistic implementation of this idea:

(4) If an utterance of " $s$ knows that $p_{1}$ " is true in context $c_{1}$ with relevant alternatives $R$, and $p_{1}$ entails $p_{2}$, then an utterance of " $s$ knows that $p_{2}$ " is true in any context $c_{2}$ with relevant alternatives $R$.

In short, the truth of knowledge ascriptions extends under entailment, as long as the relevant alternatives hold fixed. ${ }^{8}$

While contextualists are typically silent about the knowledge relation, they are perhaps best understood as epistemic pluralists. ${ }^{9}$ For the contextualist, "knows" is an indexical that ranges over a plurality of epistemic relations $\mathrm{K}_{R}$. Each set of relevant alternatives $R$ is paired with an epistemic relation $\mathrm{K}_{R}$. So 4 can be expressed in the object-language as: 
(5)

$$
\left(\mathrm{K}_{R} s p_{1} \&\left(p_{1} \rightarrow p_{2}\right)\right) \rightarrow \mathrm{K}_{R} s p_{2}
$$

In words: if $s$ knows $_{R}$ that $p_{1}$, and $p_{1}$ entails $p_{2}$, then $s$ $\operatorname{knows}_{R}$ that $p_{2}$.

Of course, 5 fails C4-C6. The omniscience, lack-of-belief, and irrational-basing problems now arise for each $\mathrm{K}_{R}$ relation. But 3 reveals how to solve these problems. So putting 3 and 5 together, and continuing on in the object-language, yields the best contextualist closure schema:

$$
\left(\mathrm{K}_{R} s p_{1} \&\left(p_{1} \rightarrow p_{2}\right)\right) \rightarrow \mathrm{E}_{R} s p_{2}
$$

That is, position to know $_{R}$ is closed under entailment. ${ }^{10}$

What can be said in favor of 6 is that it satisfies $\mathrm{C} 1-\mathrm{C} 7$ and C9. $\mathrm{C} 1$ is satisfied insofar as the intuitively compelling idea of closure is preserved, in that all epistemic relations extend under entailment. $\mathrm{C} 2$ is satisfied since proof extends all epistemic relations. C3 is satisfied since abominable conjunctions are blocked (there is no single epistemic relation $\mathrm{K}_{R}$ for which the abominable conjunction holds. C4-C6 are satisfied by borrowing the positioning maneuver from 3. C7 is satisfied since it is trivial to translate an indexed plurality of binary relations $\mathrm{K}_{R} s p$, into the contrastive relation $\mathrm{K} s p q$, by taking $q$ to be the disjunction of the members of $R$. And C9 is satisfied as long as the content of the whether-question is permitted to set the relevant alternatives. ${ }^{11}$ This yields Moore's knowing \{stumps\} $_{\text {that }}$ the has hands and his not knowing \{vat $\}$ that he has hands, which are distinct epistemic relations, and so not forced into conflict by 6 . Thus the contextualist can claim to skirt skeptical paradox.

But what can be said against 6 is that it fails $\mathrm{C} 8$, and that it is insufficient for many cases of knowledge expansion under deduction. Starting with C8, suppose that Moore knows $_{R}$ that he has hands. Then by 6 , he is in position to $\mathrm{know}_{R}$ that he is not a brain-in-a-vat. Thus all the $\mathrm{K}_{R}$ relations are either dogmatic or skeptical - none are modest. ${ }^{12}$ Further, the dogmatic epistemic relations seem to involve 'cheap knowledge' without any evidential basis. Suppose, for instance, that Moore knows $_{R}$ that he has hands, where $R=$ that Moore has stumps . To achieve this state, Moore must eliminate the alternative of 
having stumps. But now consider Moore's alleged state of knowing $_{R}$ that he is not a brain-in-a-vat. Not-being-a-brain-ina-vat is no longer an alternative to having stumps (rather, it is a super-case of it). There is no relevant alternative to not-bringa-brain-in-a-vat in $R$. So to achieve this alleged state, it seems that Moore need not eliminate anything. But surely epistemic achievements require an evidential basis. ${ }^{13}$

6 is also insufficient for many cases of knowledge expansion under deduction. As a first example, suppose I know that the murderer is Scarlet rather than Peacock or Mustard. Then I should be in position to know that the murderer is Scarlet rather than Peacock. (Equivalently in this context: suppose I know whether the murderer is Scarlet or Peacock or Mustard, then I should be in position to know whether the murderer is Scarlet or Peacock.) After all, if I can eliminate both the Peacock and Mustard alternatives, surely I can eliminate only the Peacock alternative. In general, one should be able to extend knowledge $_{R 1}$ that $p$, to knowledge $R 2$ that $p$, where $R 1 \supset R 2$. But 6 does not apply because $R$ varies. My knowledge Peacock, $_{\text {Pech }}$ Mustard that the murderer is Scarlet is disconnected from any knowledge Peacock $_{\text {P }}$ that the murderer is Scarlet.

As a second example of the insufficiency of 6, suppose I know that the murderer is Scarlet or Peacock, rather than Mustard, and that I know that the murderer is Scarlet or Green, rather than Mustard. Then I should be in position to know that the murderer is Scarlet rather than Mustard. (Equivalently in this context: suppose I know whether the murderer is (i) Scarlet or Green, or (ii) Mustard, and that I know whether the murderer is (i) Scarlet or Peacock, or (ii) Mustard; then I should be in position to know whether the murderer is Scarlet or Mustard.) After all, I can eliminate the Mustard possibility throughout. In general, one should be able to combine knowledge $e_{R}$ that $p_{1}$, with knowledge ${ }_{R}$ that $p_{2}$, to know ${ }_{R}$ that $p_{1}$ and $p_{2}$. But 6 does not apply because neither $p_{1}$ nor $p_{2}$ individually entails $p_{1}$ and $p_{2}-6$ has no mechanism for multi-premise closure. ${ }^{14}$

As a third example of the insufficiency of 6, suppose I know that the murderer is Scarlet rather than Peacock, and that I 
know that the murderer is Scarlet rather than Mustard. Then I should be in a position to know that the murderer is Scarlet rather than Peacock or Mustard. (Equivalently in this context: suppose I know whether the murderer is Scarlet or Peacock, and that I know whether the murderer is Scarlet or Mustard; then I should be in a position to know whether the murderer is Scarlet or Peacock or Mustard.) After all, if I can eliminate both of the Peacock and Mustard disjuncts individually, surely I can eliminate their disjunction. In general, one should be able to combine knowledge $\mathrm{e}_{R I}$ that $p$, with knowledge $\mathrm{e}_{R 2}$ that $p$, to know $R I \cup R 2$ that $p$. But 6 does not apply because $R$ varies, and also because there are multiple premises.

These last three points yield three further adequacy conditions:

(C10) An adequate closure schema should allow extension from knowledge $e_{R I}$ that $p$, to the position to know $_{R 2}$ that $p$, where $R l \subset R 2$.

(C11) An adequate closure schema should allow extension from knowledge $e_{R}$ that $p_{1}$ and knowledge $e_{R}$ that $p_{2}$, to the position to $\mathrm{know}_{R}$ that $p_{1}$ and $p_{2}$.

(C12) An adequate closure schema should allow extension from knowledge $e_{R I}$ that $p$ and knowledge $e_{R 2}$ that $p$, to the position to $\mathrm{know}_{R l \cup R 2}$ that $p$.

What has gone wrong is that the contextualist has added a parameter - the $R$-subscripts - but has only implemented a closure schema built for $\mathrm{Ksp} .{ }^{15}$ To fail $\mathrm{C} 10-\mathrm{C} 12$ is to be insufficient to cover deductive extensions of knowledge.

\section{KNOWLEDGE AND ANSWERS}

So how should the contrastivist formulate closure, to satisfy C1-C12? To begin, it will prove helpful to connect contrastive knowledge with the ability to answer questions. There are three main points of connection. First, $\{p, q\}$ spans the denotation of a question. A question denotes a set of multiple alternatives - all well-formed questions are multiple-choice questions. ${ }^{16}$ The possible answers to a question form multiple 
cells in a region of logical space. Questions partition the possibilities. Thus the question of whether Moore has hands or stumps can be diagrammed as:

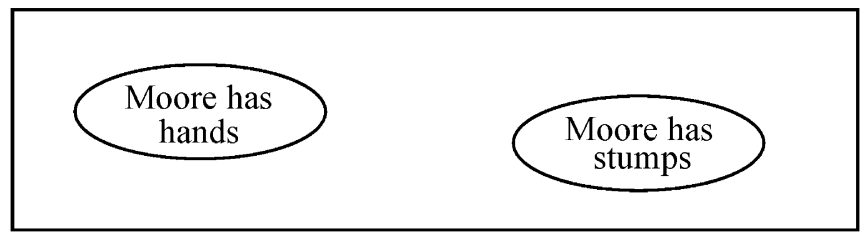

So contrastive knowledge is equivalent to question-relative knowledge: $\mathrm{K} s p q$ is equivalent to $\mathrm{K} s p Q$, where $Q$ is the question ?:\{p,q\}. For instance, Moore's knowledge that he has hands rather than stumps is equivalent to his knowledge that he has hands relative to the question of whether he has hands or stumps. ${ }^{17}$

A second point of connection between contrastive knowing and answering is that the contrasts, when left implicit, are determined by the question under discussion. A context can be modeled as a set of alternatives - those alternatives that are the 'live options' under discussion. ${ }^{18}$ So when a knowledge ascription is inexplicit as to the value of $q, q$ defaults to the disjunction of non- $p$ options under discussion. ${ }^{19}$

A third point of connection between contrastive knowing and answering is that the elimination of the relevant alternatives constitutes the ability to answer. That is, as per A1, the evidential component of contrastive knowledge is the elimination of the alternatives. Which is what it takes to be able to answer. After all, imagine facing a multiple-choice question, and eliminating all but one option. One would be in an ideal position to answer. ${ }^{20}$ Thus contrastivism implements Castañeda's idea that, "knowledge involves essentially the non-doxastic component of a power to answer a question" (1980, p. 194). All knowing is knowing the answer.

So contrastive knowledge can be represented via cells in a region of logical space, with all but one cell crossed out. So the following represents the evidential basis for Moore's knowledge that he has hands rather than stumps: 


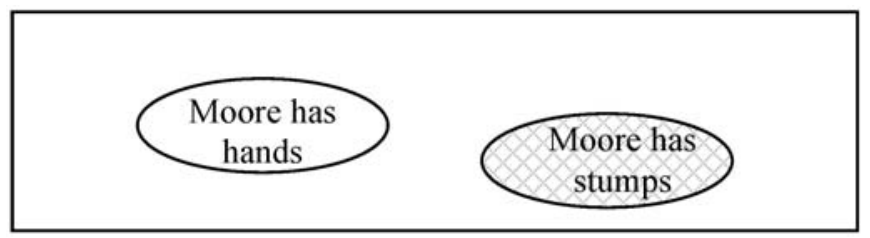

Thus the question of how the contrastivist should formulate closure may be usefully transformed into the question of how the ability to answer questions extends.

\section{EXTENDING ANSWERS}

So how does the ability to answer questions extend? Suppose that $s$ is able to answer $Q_{1}=?:\left\{p_{1}, q_{1}, q_{2}\right\}$, via $p_{1}$ :

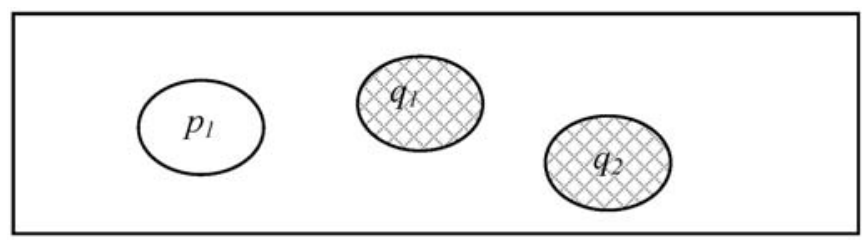

Then what other questions is $s$ is in a position to answer?

First, consider the question $Q_{2}=?:\left\{p_{1} \vee p_{2}, q_{1}, q_{2}\right\}$, where $p_{2}$ concerns a hitherto irrelevant region of logical space:
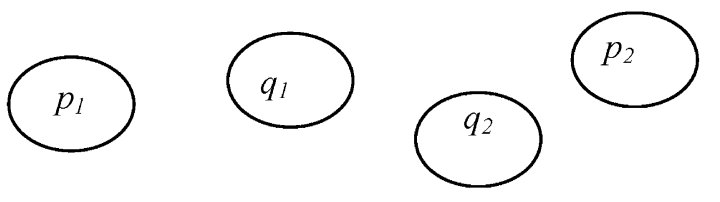

Since we are given that $s$ is able to eliminate $q_{1}$ and $q_{2}, s$ is in the position represented by:

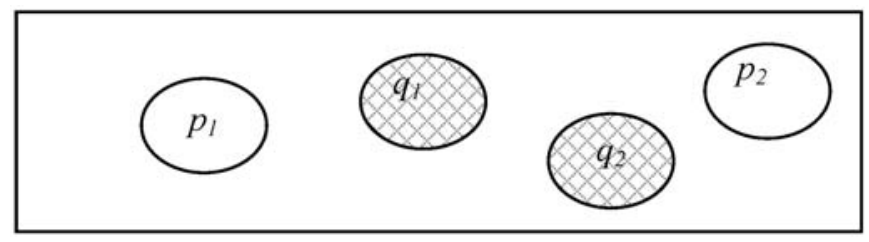


This shows that $s$ is able to answer $Q_{2}$ via $p_{1} \vee p_{2}$.

Second, consider the alleged question $Q_{3}=$ ?:\{ $\left\{p_{1} \vee \sim p_{1}, q_{1}\right.$, $\left.q_{2}\right\}$. Here the first 'cell' $\left\{p_{1} \vee \sim p_{1}\right\}$ covers the others. So this is not a genuine question, since a genuine question requires a partition. Thus $s$ is not able to answer $Q_{3}$ via $p_{1} \vee \sim p_{1}$, since $Q_{3}$ is not a genuine question that can be answered.

What the extension from $Q_{1}$ to $Q_{2}$ shows is that $\mathrm{K} s p q$ extends under $p$-expansion. This has a topological rationale, which is that any super-region of an uneliminated region is an uneliminated region. ${ }^{21}$ What the failure of extension from $Q_{1}$ to $Q_{3}$ shows is that extension under $p$-expansion is limited by $q$. If $p$ expands to overlap $q$ at any point, then what results is no longer a genuine question, and so no longer something capable of being answered. ${ }^{22}$ Putting this together yields a first closure schema:

$($ Expand- $p)\left(\mathrm{K} s_{1} p_{1} q \&\left(p_{1} \rightarrow p_{2}\right) \&\left\{p_{2}\right\} \cap\{q\}=\varnothing\right) \rightarrow \mathrm{Esp}_{2} q$ In short, position to know is closed under $q$-bounded expansion at $p$.

Third, consider the question $Q_{4}=\left\{p_{1}, q_{1}\right\}$ :

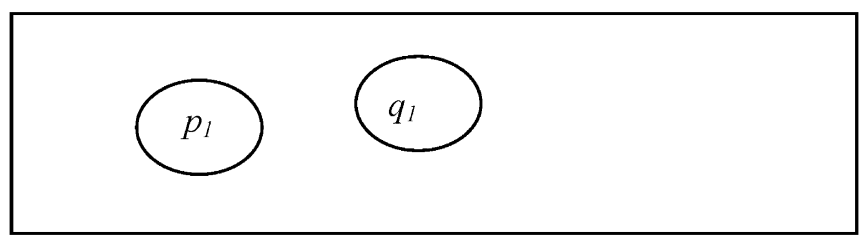

Given that $s$ is able to answer $Q 1, s$ can eliminate $q_{1}$, so $s$ is in the position represented by:

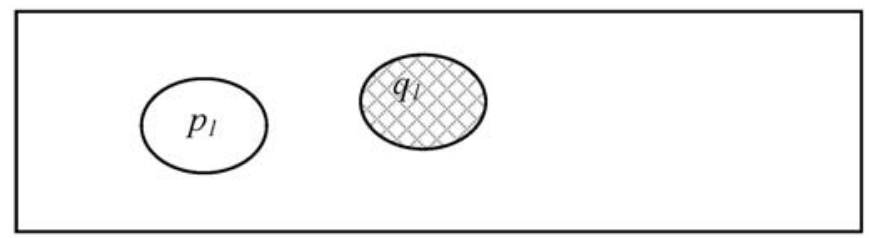

This shows that $s$ is able to answer $Q_{4}$ via $p_{1}$.

Fourth, consider the alleged question $Q_{5}=?:\left\{p_{1}\right\} . Q_{5}$ has only one cell. So $Q_{5}$ is not a genuine question, since a genuine 
question requires multiple cells. ${ }^{23}$ So $s$ is not able to answer $Q_{5}$ via $p_{1}$, since $Q_{5}$ is not a genuine question that can be answered.

What the extension from $Q_{1}$ to $Q_{4}$ shows is that $\mathrm{K} s p q$ extends under $q$-contraction. There is a topological rationale for this, which is that any subregion of an eliminated region is an eliminated region. ${ }^{24}$ What the failure of extension from $Q_{1}$ to $Q_{5}$ shows is that extension under $q$-contraction is limited by $\varnothing$. If $q$ contracts to nothing, then what results is no longer a genuine question, and so no longer something capable of being answered. Putting this together yields a second closure schema:

$($ Contract- $q)\left(\mathrm{K} s p q_{1} \&\left(q_{2} \rightarrow q_{1}\right) \&\left\{q_{2}\right\} \neq \varnothing\right) \rightarrow{\mathrm{E} s p q_{2}}$

In short, position to know is closed under nonempty contraction at $q$.

\section{COMBINING ANSWERS}

So far, Expand- $p$ and Contract- $q$ describe how knowing a single answer extends. It remains to describe how knowing multiple answers combines. Here it will suffice to consider the case of knowing two answers, since all (finite) multiplicities can be accessed by repeated application of pairwise principles.

So first, suppose that $s$ is knowingly able to answer $Q_{1}$ $\left(Q_{1}=\right.$ ?: $\left.\left\{p_{1}, q_{1}, q_{2}\right\}\right)$ via $p_{1}$, and that $s$ is knowingly able to answer $Q_{6}\left(Q_{6}=?:\left\{p_{2}, q_{1}, q_{2}\right\}\right)$ via $p_{2}$. From these suppositions and the factivity of knowledge $(\mathrm{K} s p q \rightarrow p)$, it follows that $\left\{p_{1}\right\} \cap\left\{p_{2}\right\} \neq \varnothing .^{25}$ And it follows that $\left\{p_{1}\right\} \cap\left\{p_{2}\right\}$ is an uneliminated region. ${ }^{26}$ So $s$ is in the position represented by: ${ }^{27}$

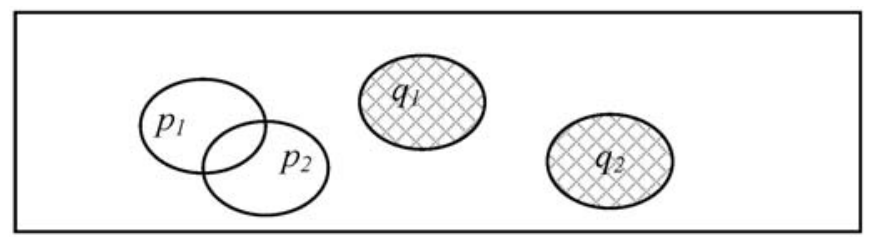

This shows that $s$ is able to answer $Q_{7}\left(Q_{7}=\right.$ ?: $\left\{p_{1} \&\right.$ $\left.\left.p_{2}, q_{1}, q_{2}\right\}\right)$ via $p_{1} \& p_{2}$. 
What the extension from $Q_{1}$ and $Q_{6}$ to $Q_{7}$ shows is that $\mathrm{K} s p_{1} q$ and $\mathrm{K} s p_{2} q$ states extend under $p$-intersection. This yields a third closure schema:

$$
(\text { Intersect- } p)\left(\mathrm{K}_{s} p_{1} q \&\left(\mathrm{~K} s p_{2} q\right) \rightarrow \mathrm{E} s\left(p_{1} \& p_{2}\right) q\right.
$$

In short, combined position to know is closed under intersection at $p$.

Second, suppose that $s$ is able to answer $Q_{4}\left(Q_{4}=?:\left\{p_{1}, q_{1}\right\}\right)$ via $p_{1}$, and that $s$ is able to answer $Q_{8}\left(Q_{8}=?:\left\{p_{1}, q_{2}\right\}\right)$ via $p_{1}$. Then by being able to answer $Q_{4}, s$ is in the following position:
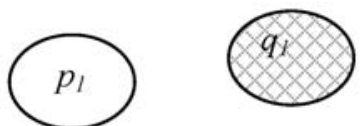

And by being able to answer $Q_{8}, s$ is in the following position:
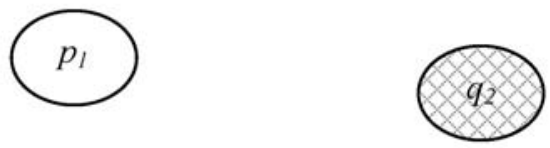

Now consider whether $s$ is able to answer $Q_{1}\left(Q_{1}=\right.$ ?: $\left.\left\{p_{1}, q_{1}, q_{2}\right\}\right)$ represented as:
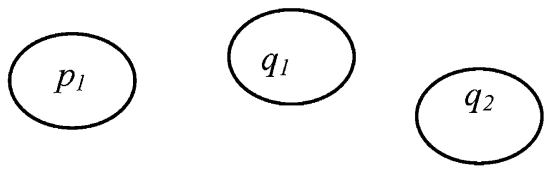

Since $s$ is able to answer $Q_{4}$, it follows that $s$ can eliminate $q_{1}$. And since $s$ is able to answer $Q_{8}$, it follows that $s$ can eliminate $q_{2}$. So $s$ is in position to eliminate both: 


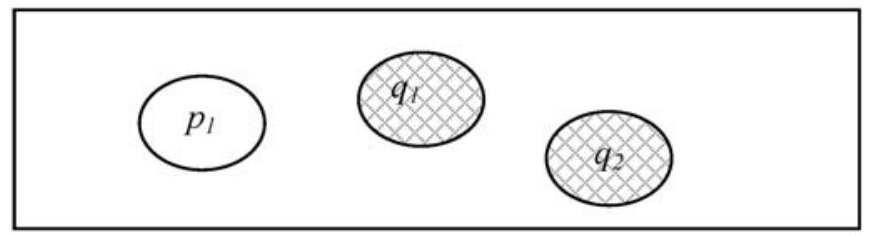

Which is what it takes to be able to answer $Q_{1}$ via $p_{1}$.

What the extension from $Q_{4}$ and $Q_{8}$ to $Q_{1}$ shows is that the $\mathrm{K} s p q_{1}$ and $\mathrm{K} s p q_{2}$ states extend under $q$-union. This yields a fourth closure schema:

$(\mathrm{Union}-q)\left(\mathrm{K} s p q_{1} \& \mathrm{~K} s p q_{2}\right) \rightarrow \operatorname{Esp}\left(q_{1} \vee q_{2}\right)$.

In short, combined position to know is closed under union at $q$.

\section{CONTRASTIVE CLOSURE}

So far I have presented 12 adequacy conditions on closure, and developed four closure schemata. Conjoining these closure schemata yields contrastive closure:

(7) Contrastive knowledge extends by Expand- $p$, Contract$q$, Intersect- $p$, and Union- $q$.

It remains to show that 7 satisfies $\mathrm{C} 1-\mathrm{C} 12$.

Starting with $\mathrm{C} 1-\mathrm{C} 3,7$ preserves the closure intuitions. A plausible rendering of how knowledge extends under entailment is provided, as per $\mathrm{C} 1$, given the equation of contrastive knowledge with the ability to answer the question ( $(4)$, and a plausible treatment of how the ability to answer questions extends (\$§5-6). Mathematical proof is allowed to extend knowledge, as per $\mathrm{C} 2$, since a proof can be regarded as extending the ability to answer questions. To take a trivial example, suppose I know that $2+2=4$ rather than any other natural number, and that I prove that $2+2=4$ entails that $2+2=3+1$. Then by Expand- $p$ I am in position to know that $2+2=3+1$ rather than any other natural number. ${ }^{28}$ Abominable conjunctions are blocked, as per $\mathrm{C} 3$, since there is no single value of $q$ that can vindicate: " $s$ does not know that she is not a brain-in-a-vat [rather than $q$ ], 
but $s$ does not that she has hands [rather than $q$ ]." For the second conjunct requires that $q$ be eliminable, and the first conjunct requires that $q$ contrast with not being a brain-in-avat. So $q$ would need to be an eliminable case of being a brain-in-a-vat. Thus there is no such $q$.

Turning to $\mathrm{C} 4-\mathrm{C} 6,7$ avoids the counterexamples. By conjoining principles with Espq consequents, 7 draws on the maneuver that avoids the counterexamples (\$2). Lack of omniscience, belief-failure, and basing-failure cannot imperil any component of 7 .

Moving to C7-C9, 7 skirts skeptical paradox. 7 fits contrastivism directly, as per C7. Epistemic modesty is preserved, as per C8. By restricting Expand- $p$ so that what is entailed cannot overlap the contrasts, cases of 'cheap knowledge' with nothing to eliminate are blocked. Thus one cannot use one's knowledge that one has hands rather than stumps, to come to know that one is not a brain-in-a-vat rather than a creature with stumps. ${ }^{29}$ And since there is no rule of Replace- $q$, one cannot use one's knowledge that one has hands rather than stumps $\left(\mathrm{K} s p q_{1}\right)$, to come to know that one has hands rather than vat-images of hands $\left(\mathrm{K} s p q_{2}\right) .{ }^{30}$ So Moore knows that he has hands rather than stumps, and does not know that he has hands rather than vat-images of hands. And knowledge-whether is accommodated, as per C9. Moore knows whether he has hands or stumps, and does not know whether he has hands or vat-images of hands. ${ }^{31}$

Finishing with $\mathrm{C} 10-\mathrm{C} 12,7$ licenses the needed inferences. C10 requires the extension from knowledge ${ }_{R 1}$ that $p$, to the position to $\mathrm{know}_{R 2}$ that $p$, where $R l \supset R 2$. This is what Contract- $q$ licenses. So if I know that the murderer is Scarlet rather than Peacock or Mustard, then, by Contract- $q$, I am in position to know that the murderer is Scarlet rather than Peacock. C11 requires the extension from knowledge ${ }_{R}$ that $p_{1}$ and knowledge $e_{R}$ that $p_{2}$, to the position to $\mathrm{know}_{R}$ that $p_{1} \&$ $p_{2}$. This is what Intersect- $p$ licenses. So if I know that the murderer is Scarlet or Peacock, rather than Mustard, and I know that the murderer is Scarlet or Green, rather than Mustard, then, by Intersect- $p$, I am in position to know that the 
murderer is Scarlet rather than Mustard. C12 requires the extension from knowledge $\mathrm{e}_{R 1}$ that $p$ and knowledge $\mathrm{e}_{R 2}$ that $p$, to the position to $\mathrm{know}_{R 1 \cup R 2}$ that $p$. This is what Union- $q$ licenses. So if I know that the murderer is Scarlet rather than Peacock, and I know that the murderer is Scarlet rather than Mustard, then I am in a position to know that the murderer is Scarlet rather than Peacock or Mustard.

No doubt there are further counterexamples to be fielded, and further inferences to be supported. Still I would conclude that contrastivism delivers the best account of closure to date. $^{32}$

\section{NOTES}

1 The contrastive theory emerges in the following passage from Dretske: "To know that $x$ is $A$ is to know that $x$ is $A$ within a framework of relevant alternatives, $B, C$, and $D$. This set of contrasts ... serve to define what it is that is known..." (1970, p. 1022) The theory has since been defended by Johnsen (2001), Morton and Karjalainen (2003), SinnottArmstrong (2004), and Schaffer (2004a, 2005b). In this vein, Johnsen maintains: "what is known is always a contrastive proposition to the effect that P-rather-than-any-other-member-of-category-C is true..." (2001, p. 401).

2 Dretske (1970), Nozick (1981), and Heller (1999a) have argued against any form of closure. I will not be addressing their arguments.

3 Hintikka (1962) treats epistemic logic via the S4 axioms:

$\mathrm{D}: \mathrm{K} s\left(p_{1} \rightarrow p_{2}\right) \rightarrow\left(\mathrm{K} s p_{1} \rightarrow \mathrm{K} s p_{2}\right)$

$\mathrm{T}: \mathrm{K} s p \rightarrow p$

$\mathrm{B}: \mathrm{K} s p \rightarrow \mathrm{KK} s p$

2 follows from D. (Proof: suppose $\mathrm{K} s p_{1}$ and $\mathrm{K} s\left(p_{1} \rightarrow p_{2}\right.$ ). From $\mathrm{K} s\left(p_{1} \rightarrow p_{2}\right)$ and $\mathrm{D}$, we get $\mathrm{K} s p_{1} \rightarrow \mathrm{K} s p_{2}$. From this and $\mathrm{K} s p_{1}$ we get $\mathrm{K} s p_{2}$. So $\left(\mathrm{K} s p_{1} \& \mathrm{~K} s\left(p_{1} \rightarrow p_{2}\right)\right) \rightarrow \mathrm{K} s p_{2}$.) Thus 2 is a consequence of modeling epistemic logic as a normal modal system, for which the distribution axioms hold.

4 Complication: $\mathrm{C} 3$ is not perfectly satisfied with 2 (and likewise for all subsequent schemata), since one can fail to know that one is not a brainin-a-vat and still know that one has hands, if one does not see the entailment relation here. But this is the right result. Really the conjunction is only abominable for the subject who sees the connection between handedness and not being envatted. The abominable conjunction can hold true of abominable reasoners. 
5 The idea of 'position to know' is invoked in Cohen $(1999$, p. 84). The understanding of position-to-know as only needing a belief-based-on-competent-deduction-while-retaining-knowledge-of-the-premises in order to know, is drawn from Williamson (2000) and Hawthorne (2004). This represents the state-of-the-art for binary closure.

6 Dretske rejects closure for essentially this reason. According to Dretske, one should be able to know that the animal is a zebra, without being in position to know that the animal is not a cleverly painted mule. Of course, most binary theorists will have already accustomed themselves to accepting that one can know that one is not a brain-in-a-vat, and that one can know that the animal is not a cleverly painted mule. But I think this should be reckoned a counterintuitive consequence.

7 For instance, Lewis writes: "Holmes knows whether ... if and only if he knows the true one of the alternatives presented by the 'whether'clause, whichever one that is." (1998, p. 45) This is an instance of the standard binary view of knowledge- $w$ h constructions. Here Higginbotham provides the rule: "know $(x, \lambda) \leftrightarrow(\exists p)(\operatorname{know}(x, p) \& p$ answers $\pi)$ " (1982, p. 194). See Schaffer (forthcoming) for further discussion.

8 The idea of closure for fixed alternatives is due to Stine (1976), and has been upheld by leading contextualists since, including Cohen (1988), DeRose (1995), and Lewis (1996).

9 Heller is explicit here: "What contextualism tell us is that 'knowledge' refers to different properties in different contexts." (1999b, p. 115) And: "Every selection of worlds defines a specific epistemic property. It is these various properties that are the possible referents for the term 'knowledge.." (1999b, p. 117) See Schaffer 2004b (\$2) for further discussion.

10 The understanding of contextualist closure via 6 is contentious in two main respects. First, not all contextualists will want to index to relevant alternatives. Some contextualists, such as Cohen 1999, will prefer to index to epistemic standards (which concern the degree of evidence sufficient to satisfy 'justified'). So this sort of contextualist might replace 6 with:

$(6 s)\left(\mathrm{K}_{s} s p_{1} \&\left(p_{1} \rightarrow p_{2}\right)\right) \rightarrow \mathrm{K}_{s} s p_{2}$

Where $S$ denotes an epistemic standard. 6s will function roughly equivalently to 6 from the perspective of the adequacy conditions of the main text, save that it will render the satisfaction of C9 more mysterious (see footnote 11). In the main text I will continue to presume that the contextualist is a relevant alternatives theorist. For arguments in favor of the relevant alternatives style of contextualism, see Schaffer (2005a).

The second respect in which the understanding of contextualism in 6 is contentious is that some contextualists (Cohen, 1999, p. 61) will also count ternary theories (including contrastivism) as forms of "contextualism'. So this sort of theorist might count the following as a variant form of 'contextualism': 
$(6 t)\left(\mathrm{K} s p_{1} \mathrm{R} \&\left(p_{1} \rightarrow p_{2}\right)\right) \rightarrow \mathrm{K} s p_{2} \mathrm{R}$

By treating the contrast proposition $q$ as the disjunction of the relevant alternatives in $R$, one can see that $6 \mathrm{t}$ is equivalent to the following contrastive schema (which is a simplified version of Expand- $p$ : §5):

$(6 c)\left(\mathrm{K} s p_{1} q \&\left(p_{1} \rightarrow p_{2}\right)\right) \rightarrow \mathrm{K} s p_{2} q$

Or, replacing relevant alternatives in $6 \mathrm{t}$ with standards:

$(6 t s)\left(\mathrm{K} s p_{1} \mathrm{~S} \&\left(p_{1} \rightarrow p_{2}\right)\right) \rightarrow \mathrm{K} s p_{2} \mathrm{~S}$

The issue of what counts as 'contextualism' here is purely terminological. I am interested in distinguishing between (i) the pluralist view that there are many binary $\mathrm{K}$ relations (in 6 and $6 \mathrm{~s}$ ), and (ii) the ternarist view that there is only one ternary $\mathrm{K}$ relation (in $6 \mathrm{t}, 6 \mathrm{c}$, and $6 \mathrm{ts}$ ). I am using 'contextualism' to cover (i) and reserving 'contrastivism' to cover the case of (ii) where the third argument place is a contrast slot. The philosopher who prefers a more general construal of 'contextualism' is welcome to regard all these theories as species of 'contextualism' (she might then distinguish 'pluralist contextualism' from 'contrastivist contextualism'). I only ask that she mark the distinction here, in whatever terminology she prefers.

11 Though it is not obvious that the contextualist is entitled to this. For instance, if the contextualist follows anything like the relevance rules of Lewis (1996), then what her rules deliver will not match what the whether-clause queries (Schaffer, 2004a). While if the contextualist prefers to index to epistemic standards (see footnote 10), then she will need to forge a connection between the alternatives of the whether-question and the standards. It is not at all obvious how this would go. I will waive this concern in the main text.

12 The best the contextualist can do is to rig the rules of relevance to create the appearance of modesty, by having all assertions of modest knowledge set the context to a dogmatic epistemic relation $\mathrm{K}_{R 1}$, and all assertions of modest ignorance set the context to a skeptical epistemic relation $\mathrm{K}_{R 2}$.

13 Here I am drawing on Heller (1999a) and Kvanvig (manuscript). For Heller, the resulting 'cheap knowledge' constitutes a reason to discard closure entirely. For Kvanvig, it constitutes a decisive objection to contextualism and contrastivism alike. Kvanvig gives the case where $s$ knows that Fido is a dog rather than a cat, claims that closure should allow $s$ to know that Fido is an animal rather than a cat, and charges that the contrastivist can make no sense of this. My reply is that contrastive closure (as formulated below: §7) in fact does not allow $s$ to know that Fido is an animal rather than a cat. This is the right result, 
since such a state is indeed nonsensical: being an animal does not contrast with being a cat.

14 Here I am adding the assumption that multi-premise closure should hold. I take the example in the main text to illustrate the plausibility of this assumption. For further defense see Hawthorne (2004, pp. 46-50).

15 The problem is that the $R$-subscripts are blocking what would otherwise be valid inferences. That is, the inferences in $\mathrm{C} 10-\mathrm{C} 12$ are valid under the binary closure schema 3. With 3, C10 becomes the valid inference from $\mathrm{K} s p$ to $\mathrm{Esp}$; $\mathrm{C} 11$ becomes the valid multi-premise inference from ( $\mathrm{K} s p \& \mathrm{~K} s q)$ to $\mathrm{E} s(p \& q)$; and $\mathrm{C} 12$ becomes the valid inference from (Ksp \& Ksp) to Esp. Thus 6 cannot do the work that 3 does. The contextualist needs to regain these lost inferences.

16 The association of questions with multiple-choice slates is known as Hamblin's dictum (Hamblin, 1958), and is implemented in Belnap and Steel's (1976) erotetic logic, and maintained in the partition semantics of Groenendijk and Stokhof (1997).

17 If the question $Q$ has more than two answers, $\mathrm{K} s p Q$ contains more information than $\mathrm{Kspq}$, since $Q$ partitions the contrasts, while $q$ lumps them into one big disjunction. But this additional information plays no epistemic role. All the alternatives must be eliminated, however they are partitioned.

18 The strategy of modeling contexts in terms of sets of alternatives is due to Stalnaker (1999a, b). For general linguistic evidence that the conversational score includes an entry for question under discussion, see Levinson (1983) and Ginzburg (1996).

19 When a knowledge ascription is explicit as to the value of $q$, via such mechanisms as explicit "rather than"-clauses, interrogative complements (including knowledge-whether constructions), or contrastive stress, $q$ is to be set to its explicit value. Note that most knowledge ascriptions involve interrogative complements. Here are some common examples: "I know what time it is," "You know who wrote the paper," and "She knows where to go." Note also that most spoken knowledge ascriptions with declarative complements feature contrastive stress. Thus compare: "I know that John kissed Mary," "I know that John kissed Mary," "I know that John kissed Mary." The unstressed binary form, as in "I know that John kissed Mary," thus seems relatively rare. Most ordinary knowledge ascriptions are explicitly contrastive.

20 As the referee for Philosophical Studies noted, one might also want some positive grounds for the remaining option. If so, one should take Espq to require both the elimination of $q$, and some positive grounds for $p$ (see Schaffer, 2005b, $\$ 4$ ). This would require more complicated diagrams, to encode the distinction between merely uneliminated versus positively supported cells. I would reach the same conclusions, but only given two further assumptions: (i) some positive support for a region 
entails some positive support for its super-regions, and (ii) some positive support for two compatible regions entails some positive support for their intersection. In the main text, I will work with the simpler treatment.

21 Proof: Let $p_{1}$ be an uneliminated region and let $p_{2}$ be a super-region of $p_{1}$. Since $p_{1}$ is uneliminated, there is at least one possibility $w$ in $p_{1}$ which is uneliminated. Since $p_{2}$ is a super-region of $p_{1}$, and $w$ is in $p_{1}$, it follows that $w$ is in $p_{2}$. So there is at least one possibility in $p_{2}$ which is uneliminated, namely $w$. So $p_{2}$ is an uneliminated region.

22 What if $p$ expands only to partially overlap $q$ ? Intuitively, if I know that the murderer is Scarlet rather than Peacock or Mustard, then I am in position to know that the murderer is (Scarlet or Peacock), rather than Mustard. This inference is allowed via two steps. First (as will emerge below), one can contract $q$. So if one knows that the murderer is Scarlet rather than Peacock or Mustard, one can know that the murderer is Scarlet rather than Mustard. One can then expand $p$, to know that the murderer is (Scarlet or Peacock), rather than Mustard. Note that this will only work for partial overlap (as it should). In cases where $p$ expands to swallow $q$ completely, no contraction of $q$ short of $\varnothing$ will allow for subsequent $p$ expansion without overlap. And (as per below) it is a constraint on $q$-contraction that $q$ remain nonempty.

${ }^{23} \mathrm{Q}_{5}$ is rather what is called a 'merely rhetorical question'. In only allowing one option, it does not call for an answer. Rather its discourse function is that of a (veiled) assertion.

24 Proof: Let $q_{1}$ be an eliminated region and let $q_{2}$ be a subregion of $q_{1}$. Since $q_{1}$ is eliminated, every possibility $w$ in $q_{1}$ is eliminated. Since $q_{2}$ is a subregion of $q_{1}$, every possibility in $q_{2}$ is a possibility in $q_{1}$, and is thus eliminated. So $q_{2}$ is an eliminated region.

25 Proof: Given $\operatorname{Ksp}_{1}\left(q_{1} \vee q_{2}\right)$ and factivity, it follows that $\alpha$ (the actual world $) \in\left\{p_{1}\right\}$. Likewise, given $\mathrm{Ksp}_{2}\left(q_{1} \vee q_{2}\right)$ and factivity, it follows that $\alpha \in\left\{p_{1}\right\}$. So $\alpha \in\left\{p_{1}\right\} \cap\left\{p_{2}\right\}$, so $\left\{p_{1}\right\} \cap\left\{p_{2}\right\} \neq \varnothing$.

26 Proof: From the previous proof, we have. $\alpha \in\left\{p_{1}\right\} \cap\left\{p_{2}\right\}$ But $\alpha$ is uneliminated by definition (Lewis, 1996), so there is an uneliminated possibility in $\left\{p_{1}\right\} \cap\left\{p_{2}\right\}$, so $\left\{p_{1}\right\} \cap\left\{p_{2}\right\}$ is an uneliminated region.

27 I have diagrammed the case where neither $p_{1}$ nor $p_{2}$ is a part of the other. But the same point holds if either is a part of the other. Actually the result will be trivial in that case, since the Intersect- $p$ rule (below) will output knowledge of the part, which will be the same bit of knowledge as was originally supposed. (That is, if $p_{1}$ is a part of $p_{2}$, then Intersect- $p$ will output $\mathrm{K}_{s} p_{1} q$, which is just the first supposition.)

28 Though here the possible world treatment of propositions goes awry, since mathematical propositions hold at all worlds or none. This is a problem for everyone. I take no position here on how to solve it here. 
29 Likewise for Kvanvig's example (as per footnote 13), one cannot use knowledge that Fido is a dog rather than a cat, to come to know that Fido is an animal rather than a cat. After all, "Is Fido an animal or a cat?" does not constitute a genuine question. And the state of knowing whether Fido is an animal or a cat does not constitute a genuine state.

30 Complication: $q$-expansion is permitted in the combined case, via Union- $q$. In order to use Union- $q$ to replace one's modest knowledge that one has hands rather than stumps, with immodest knowledge that one has hands rather than vat-images of hands, one would need a second piece of knowledge. This second piece of knowledge would need to be knowledge that one has hands rather than $q_{2}$, where $q_{1} \in q_{2}$ would be the brain-in-a-vat possibility (or a superset thereof, with Contract- $q$ then being applied to pare down to the brain-in-a-vat possibility). So $q_{2}$ would need to contain the brain-in-a-vat hypothesis. But that would make it ineliminable. Thus no such second piece of knowledge exists.

31 Similar comments apply to Dretske's example (as per footnote 6). One can know that the animal is a zebra rather than a normal mule, and not know that the animal is a zebra rather than a cleverly painted mule. Further, one can know whether the animal is a zebra or a normal mule, and not know whether the animal is a zebra or a painted mule.

32 Thanks to Martijn Blaauw, Stewart Cohen, Bjørn Jespersen, Jonathan Kvanvig, Ram Neta, the referee for Philosophical Studies, and the audience for the Contextualism Conference at the Free University of Amsterdam.

\section{REFERENCES}

Belnap, N. and Steel, T. (1976): The Logic of Questions and Answers, and New Haven.

Castañeda, H. (1980): 'The Theory of Questions, Epistemic Powers, and the Indexical Theory of Knowledge', in P. French, T. Uehling and H. Wettstein Jr. (eds.), Midwest Studies in Philosophy V: Studies in Epistemology, Minneapolis, 193-238.

Cohen, S. (1988): 'How to be a Fallibilist', Philosophical Perspectives 2, 91-123.

Cohen, S. (1999): 'Contextualism, Skepticism, and the Structure of Reasons', Philosophical Perspectives 13, 57-89.

DeRose, K. (1995): Solving the Skeptical Problem', Philosophical Review 104, 1-52.

Dretske, F. (1970): 'Epistemic Operators', Journal of Philosophy 67, 10071023.

Dretske, F. (1981): 'The Pragmatic Dimension of Knowledge', Philosophical Studies 40, 363-378. 
Feldman, R. (1995): 'In Defense of Closure', Philosophical Quarterly 45, 487-494.

Ginzburg, J. (1996): 'Interrogatives: Questions, Facts and Dialogue', in S. Lappin (ed.), The Handbook of Contemporary Semantic Theory (London), 385-422.

Groenendijk, J. and M. Stokhof (1997): 'Questions', in J. van Benthem and A. ter Meulen (eds.), Handbook of Logic and Language (Amsterdam), $1055-1124$.

Hamblin, C. L. (1958): 'Questions', Australasian Journal of Philosophy 36, 159-68.

Hawthorne, J. (2004): Knowledge and Lotteries, Oxford.

Heller, M. (1999a): Relevant Alternatives and Closure', Australasian Journal of Philosophy 77, 196-208.

Heller, M. (1999b): The Proper Role for Contextualism in an Anti-Luck Epistemology', Philosophical Perspectives 13, 115-129.

Higginbotham, J. (1996): 'The Semantics of Questions', in S. Lappin (ed.), The Handbook of Contemporary Semantic Theory, Oxford, pp. 361-383.

Hintikka, J. (1962): Knowledge and Belief, Ithaca.

Johnsen, B. (2001): Contextualist Swords, Skeptical Plowshares', Philosophy and Phenomenological Research 62, 385-406.

Kvanvig, J. (forthcoming): 'Contextualism, Contrastivism, Relevant Alternatives, and Closure', Philosophical Studies.

Levinson, S. (1983): Pragmatics, Cambridge.

Lewis D. (1982): 'Whether Report', in T. Pauli (ed.), Philosophical Essays Dedicated to Lennart Åqvist on his Fiftieth Birthday, Uppsala, pp. 194-206.

Lewis, D. (1996): 'Elusive Knowledge', Australasian Journal of Philosophy 74, 549-567.

Morton, A. and A. Karjalainen (2003): Contrastive Knowledge', Philosophical Explorations 6, 74-89.

Nozick, R. (1981): Philosophical Explanations, Cambridge.

Schaffer, J. (2004a): From Contextualism to Contrastivism', Philosophical Studies 119, 73-103.

Schaffer, J. (2004b): Skepticism Contextualism, and Discrimination', Philosophy Phenomenological Research 69, 138-155.

Schaffer, J. (2005a): 'What Shifts? Thresholds, Standards, or Alternatives?', in G. Preyer and G. Peter (eds.), Contextualism in Philosophy, Oxford, pp. 115-130.

Schaffer, J. (2005b): 'Contrastive Knowledge', in T. Gendler and J. Hawthorne (eds.), Oxford Studies in Epistemology 1, pp. 235-271.

Schaffer, J. (forthcoming): 'Knowing the Answer', Philosophy and Phenomenological Research.

Sinnott-Armstrong, W. (2004): Pyrrhonian Skepticism, Oxford.

Stalnaker, R. (1999a): 'Assertion', Context and Content, Oxford, pp. 78-95. 
Stalnaker, R. (1999b): 'On the Representation of Context', Context and Content, Oxford, pp. 96-113.

Stine, G. C. (1976): Skepticism Relevant Alternatives, and Deductive Closure', Philosophical Studies 29, 249-261.

Williamson, T. (2000): Knowledge and its Limits, Oxford.

Department of Philosophy

University of Massachusetts-Amherst

Amherst, MA 01003

USA

E-mail: schaffer@philos.umass.edu 